\title{
New application of sine-cosine method for the generalized $(2+1)$-dimensional nonlinear evolution equations
}

\author{
Malihe Najafi*, Somayeh Arbabi, Mohammad Najafi \\ Department of Mathematics, Anar Branch, Islamic Azad University, Anar, Iran \\ *Corresponding author E-mail: mnajafi82@gmail.com
}

\begin{abstract}
By means of the idea of sine-cosine method, some analytic solutions for the generalized $(2+1)$-dimensional nonlinear evolution equations are presented. $(2+1)$-dimensional breaking soliton equation and $(2+1)$-dimensional CalogeroBogoyavlenskii-Schiff (CBS) equation and (2+1)-dimensional Bogoyavlenskii's Breaking soliton equation are chosen to illustrate the effectiveness of the method.

Keywords: Sine-cosine method, (2+1)-dimensional CBS equation, (2+1)-dimensional Breaking soliton equation, (2+1)-dimensional Bogoyavlenskii's Breaking soliton equation.
\end{abstract}

\section{Introduction}

In this work, we will study the generalized (2+1)-dimensional nonlinear evolution equations

$$
u_{x t}+a u_{x} u_{x y}+b u_{x x} u_{y}+u_{x x x y}=0,
$$

where $a$ and $b$ are parameters. For example, namely the (2+1)-dimensional Calogero-Bogoyavlenskii-Schiff (CBS) equation for which $a=4$ and $b=2$,

$$
u_{x t}+4 u_{x} u_{x y}+2 u_{x x} u_{y}+u_{x x x y}=0,
$$

and the (2+1)-dimensional breaking soliton equation for which $a=-4$ and $b=-2$,

$$
u_{x t}-4 u_{x} u_{x y}-2 u_{x x} u_{y}+u_{x x x y}=0,
$$

and the (2+1)-dimensional Bogoyavlenskii's Breaking Soliton equation for which $a=4$ and $b=4$,

$$
u_{x t}+4 u_{x} u_{x y}+4 u_{x x} u_{y}+u_{x x x y}=0,
$$

In this paper we solve equation (1) by the sine-cosine method method and obtain some exact and new solutions for (2),(3) and (4).

\section{The Sine-cosine method}

1. We introduce the wave variable $\xi=x-c t$ into the PDE

$$
P\left(u, u_{t}, u_{x}, u_{t t}, u_{x x}, u_{t x}, \ldots\right)=0,
$$

where $u(x, t)$ is traveling wave solution. This enables us to use the following changes:

$$
\frac{\partial}{\partial t}=-c \frac{\partial}{\partial \xi}, \frac{\partial^{2}}{\partial t^{2}}=c^{2} \frac{\partial^{2}}{\partial \xi^{2}}, \frac{\partial}{\partial x}=\frac{\partial}{\partial \xi}, \frac{\partial^{2}}{\partial x^{2}}=\frac{\partial^{2}}{\partial \xi^{2}}, \ldots
$$


One can immediately reduce the nonlinear PDE (5) into a nonlinear ODE

$$
Q\left(u, u_{\xi}, u_{\xi \xi}, u_{\xi \xi \xi}, \ldots\right)=0
$$

The ordinary differential equation (7) is then integrated as long as all terms contain derivatives, where we neglect integration constants.

2. The solutions of many nonlinear equations can be expressed in the form [1]

$$
u(x, t)= \begin{cases}\lambda \sin ^{\beta}(\mu \xi), & |\xi| \leq \frac{\pi}{\mu} \\ 0 & \text { otherwise }\end{cases}
$$

or in the form

$$
u(x, t)= \begin{cases}\lambda \cos ^{\beta}(\mu \xi), & |\xi| \leq \frac{\pi}{2 \mu} \\ 0 & \text { otherwise }\end{cases}
$$

where $\lambda, \mu$ and $\beta \neq 0$ are parameters that will be determined, $\mu$ and $c$ are the wave number and the wave speed respectively. We use

$$
\begin{aligned}
& u(\xi)=\lambda \sin ^{\beta}(\mu \xi) \\
& u^{n}(\xi)=\lambda^{n} \sin ^{n \beta}(\mu \xi) \\
& \left(u^{n}\right)_{\xi}=n \mu \beta \lambda^{n} \cos (\mu \xi) \sin ^{n \beta-1}(\mu \xi), \\
& \left(u^{n}\right)_{\xi \xi}=-n^{2} \mu^{2} \beta^{2} \lambda^{n} \sin ^{n \beta}(\mu \xi)+n \mu^{2} \lambda^{n} \beta(n \beta-1) \sin ^{n \beta-2}(\mu \xi),
\end{aligned}
$$

and the derivatives of 9 becoms

$$
\begin{aligned}
& u(\xi)=\lambda \cos ^{\beta}(\mu \xi), \\
& u^{n}(\xi)=\lambda^{n} \cos ^{n \beta}(\mu \xi), \\
& \left(u^{n}\right)_{\xi}=-n \mu \beta \lambda^{n} \sin (\mu \xi) \cos ^{n \beta-1}(\mu \xi), \\
& \left(u^{n}\right)_{\xi \xi}=-n^{2} \mu^{2} \beta^{2} \lambda^{n} \cos ^{n \beta}(\mu \xi)+n \mu^{2} \lambda^{n} \beta(n \beta-1) \cos ^{n \beta-2}(\mu \xi),
\end{aligned}
$$

and so on for other derivatives.

3. We substitute (10) or (11) into the reduced equation obtained above in (7), balance the terms of the cosine functions when (11) is used, or balance the terms of the sine functions when (10) is used, and solving the resulting system of algebraic equations by using the computerized symbolic calculations. We next collect all terms whit same power in $\cos ^{k}(\mu \xi)$ or $\sin ^{k}(\mu \xi)$ and set to zero their coefficients to get a system of algebraic equations among the unknowns $\mu, \beta$ and $\lambda$. We obtained all possible value of the parameters $\mu, \beta$ and $\lambda[2]$.

\section{New application Sine-cosine method}

In this section we apply the sine-cosine method to the generalized $(2+1)$-dimensional nonlinear evolution equations

$$
u_{x t}+a u_{x} u_{x y}+b u_{x x} u_{y}+u_{x x x y}=0
$$

We use the wave transformation

$$
u(\xi)=u(x, y, t), \xi=x+y-c t,
$$

where $c$ is constant to be determined later. Substituting (13) into system (12), we obtain an ordinary differential equation:

$$
-c u^{\prime \prime}+a u^{\prime} u^{\prime \prime}+b u^{\prime} u^{\prime \prime}+u^{(4)}=0
$$


or equivalently

$$
-c u^{\prime \prime}+(a+b) u^{\prime} u^{\prime \prime}+u^{(4)}=0
$$

where prime denotes the differential with respect to $\xi$. Integrating (15) with respect to $\xi$ and taking the integration constant as zero yields

$$
-c u^{\prime}+\frac{(a+b)}{2}\left(u^{\prime}\right)^{2}+u^{(3)}=0
$$

setting $u^{\prime}(\xi)=v(\xi)$, Eq. (16) becomes

$$
-c v+\frac{(a+b)}{2} v^{2}+v^{\prime \prime}=0 .
$$

Substituting (8) into (17) gives

$$
\left.-c \lambda \sin ^{\beta}(\mu \xi)-\mu^{2} \beta^{2} \lambda \sin ^{\beta}(\mu \xi)+\mu^{2} \lambda \beta(\beta-1) \sin ^{\beta-2}(\mu \xi)\right)+\frac{a+b}{2} \lambda^{2} \sin ^{2 \beta}(\mu \xi)=0 .
$$

Equating the exponents and the coefficients of each pair of the sine functions we find the following system of algebraic equations:

$$
\begin{aligned}
& (\beta-1) \neq 0, \\
& \beta-2=2 \beta, \\
& -c \lambda-\mu^{2} \beta^{2} \lambda=0, \\
& \lambda \mu^{2} \beta(\beta-1)+\frac{a+b}{2} \lambda^{2}=0,
\end{aligned}
$$

Solving the system (19) yields

$$
\beta=-2 \quad, \quad \mu=\frac{1}{2} \sqrt{-c} \quad, \quad \lambda=\frac{3 c}{a+b},
$$

where $\mathrm{c}$ is a free parameter. Hence, for $c<0$, the following periodic solutions

$$
v_{1}(\xi)=\frac{3 c}{a+b} \csc ^{2}\left[\frac{\sqrt{-c}}{2} \xi\right]
$$

where $0<\frac{1}{2} \sqrt{-c} \xi<\pi$, and

$$
v_{2}(\xi)=\frac{3 c}{a+b} \sec ^{2}\left[\frac{\sqrt{-c}}{2} \xi\right]
$$

where $\left|\frac{1}{2} \sqrt{-c} \xi\right|<\frac{\pi}{2}$. In view of these results, and recall that $u^{\prime}(\xi)=v(\xi)$, integrating $(21)$ and $(22)$ with respect to $\xi$ and considering the zero constants for integration we obtain

$$
\begin{aligned}
& u_{1}(\xi)=-\frac{6 c}{\sqrt{-c}(a+b)} \cot \left[\frac{\sqrt{-c}}{2} \xi\right], \\
& u_{2}(\xi)=\frac{6 c}{\sqrt{-c}(a+b)} \tan \left[\frac{\sqrt{-c}}{2} \xi\right],
\end{aligned}
$$

using $u(x, y, t)=u(\xi)$ and $\xi=x+y-c t$ we get

$$
\begin{aligned}
& u_{1}(x, y, t)=-\frac{6 c}{\sqrt{-c}(a+b)} \cot \left[\frac{\sqrt{-c}}{2}(x+y-c t)\right], \\
& u_{2}(x, y, t)=\frac{6 c}{\sqrt{-c}(a+b)} \tan \left[\frac{\sqrt{-c}}{2}(x+y-c t)\right] .
\end{aligned}
$$




\section{Exact solution of $(2+1)$-dimensional CBS equation}

In this section, we investigate explicit formula of solutions of the following (2+1)-dimensional Calogero-BogoyavlenskiiSchiff (CBS) equation given in [3]

$$
u_{x t}+4 u_{x} u_{x y}+2 u_{x x} u_{y}+u_{x x x y}=0,
$$

by using section (3), we have following exact solutions:

\section{Exact solution I:}

$$
u_{1}(x, y, t)=-\frac{c}{\sqrt{-c}} \cot \left[\frac{\sqrt{-c}}{2}(x+y-c t)\right],
$$

where $0<\frac{1}{2} \sqrt{-c}(x+y-c t)<\pi$.

Exact solution II:

$$
u_{2}(x, y, t)=\frac{c}{\sqrt{-c}} \tan \left[\frac{\sqrt{-c}}{2}(x+y-c t)\right],
$$

where $\left|\frac{1}{2} \sqrt{-c}(x+y-c t)\right|<\frac{\pi}{2}$.

\section{Exact solution of (2+1)-dimensional Breaking soliton equation}

In this section, we investigate explicit formula of solutions of the following (2+1)-dimensional Breaking soliton equation given in [4]

$$
u_{x t}-4 u_{x} u_{x y}-2 u_{x x} u_{y}+u_{x x x y}=0,
$$

by using section (3), we have following exact solutions:

\section{Exact solution I:}

$$
u_{1}(x, y, t)=\frac{c}{\sqrt{-c}} \cot \left[\frac{\sqrt{-c}}{2}(x+y-c t)\right],
$$

where $0<\frac{1}{2} \sqrt{-c}(x+y-c t)<\pi$.

\section{Exact solution II:}

$$
u_{2}(x, y, t)=-\frac{c}{\sqrt{-c}} \tan \left[\frac{\sqrt{-c}}{2}(x+y-c t)\right],
$$

where $\left|\frac{1}{2} \sqrt{-c}(x+y-c t)\right|<\frac{\pi}{2}$.

\section{Exact solution of $(2+1)$-dimensional Bogoyavlenskii's Breaking soli- ton equation}

In this section, we investigate explicit formula of solutions of the following (2+1)-dimensional Bogoyavlenskii's Breaking soliton equation given in [5]

$$
u_{x t}+4 u_{x} u_{x y}+4 u_{x x} u_{y}+u_{x x x y}=0,
$$

by using section (3), we have following exact solutions: 


\section{Exact solution I:}

$$
u_{1}(x, y, t)=\frac{3 c}{4 \sqrt{-c}} \cot \left[\frac{\sqrt{-c}}{2}(x+y-c t)\right]
$$

where $0<\frac{1}{2} \sqrt{-c}(x+y-c t)<\pi$.

\section{Exact solution II:}

$$
u_{2}(x, y, t)=-\frac{3 c}{4 \sqrt{-c}} \tan \left[\frac{\sqrt{-c}}{2}(x+y-c t)\right],
$$

where $\left|\frac{1}{2} \sqrt{-c}(x+y-c t)\right|<\frac{\pi}{2}$.

\section{Conclusion}

In this paper, by using the sine-cosine method, we obtained some explicit formulas of solutions for the generalized $(2+1)$-dimensional nonlinear evolution equations. Those solutions were similar to the solutions obtained in other paper. The study reveals the power of the method.

\section{References}

[1] A.M. Wazwaz, The sine-cosine method for obtaining solutions with compact and noncompact structures, Appl. Math. Comput. 159 (2) (2004) 559-576.

[2] A.M. Wazwaz, A sine-cosine method for handling nonlinear wave equations, Math. Comput. Model. 40 (2004) $499-508$.

[3] A.M. Wazwaz, The $(2+1)$ and (3+1)-Dimensional CBS Equations: Multiple Soliton Solutions and Multiple Singular Soliton Solutions, Zeitschrift fur Naturforschung A, 65a (2010 )173-181.

[4] A.M. Wazwaz, Integrable (2+1)-dimensional and (3+1)-dimensional breaking soliton equations, Phys. Scr. 81 (2010) 1-5.

[5] M.T. Darvishi, M. Najafi, Some exact solutions of the (2+1)-dimensional breaking soliton equation using the three-wave method, International Journal of Computational and Mathematical Sciences 6 (2012) 13-16. 